\title{
Performance Evaluation of Spectrum Sharing Methods in WiMAX/WiFi Integrated Networks
}

\author{
Mohammed Nowaz Rabbani Chowdhury ${ }^{1}$, Jannatul Mawa ${ }^{2}$ and Subrata Kumar Aditya ${ }^{2}$ \\ ${ }^{1}$. Department of Electronic and Telecommunication Engineering, Daffodil International University, \\ Dhanmondi, Dhaka-1207, Bangladesh. \\ ${ }^{2}$. Department of Electrical and Electronic Engineering, Dhaka University, Dhaka-1000, Bangladesh
}

(Received: 15 January 2018; Accepted: 5 March 2018)

\begin{abstract}
Next generation wireless communication system is influenced by different wireless technologies such as WiFi, WiMAX, Cellular and LTE which use their fixed allocated spectrum. However, lack of spectrum resources becomes an important problem for future wireless network. Hence, for efficient use of spectrum resources, spectrum sharing between the existing wireless technologies receives much attention. However, to share the spectrum more efficiently, an effective spectrum sharing method should be developed. Most of the spectrum sharing methods are based on an optimization algorithm that provides an optimal solution for spectrum sharing. In this paper, the performance of a spectrum sharing method based on Genetic Algorithm (GA) has been evaluated. The method was applied in a WiMAX/WiFi integrated network, where a WiFi system temporarily uses a spectrum band of WiMAX system. Also a Linear Programming (LP) model has been developed as an alternative for spectrum sharing in the same network and comparative analysis between these two methods reveals that both methods give better performance in terms of throughput (bits/sec), download response time (sec) and received traffic rate (bytes/sec). According to the simulation results, it has been found that Genetic Algorithm (GA) shows its effectiveness in spectrum sharing in WiMAX/WiFi integrated network by reaching the global maxima very fast.
\end{abstract}

Keywords: Integrated networks, WiMAX, WiFi, Linear Programming, Genetic Algorithm.

\section{Introduction}

Advanced communication technologies provide various diversified network services via internet. People want to use these services not only via wired networks but also via wireless networks. Hence the demand for the mobile wireless services continues to explode ${ }^{1}$.

Moreover, there are several wireless access technologies such as cellular, WiFi, WiMAX and $\mathrm{LTE}^{2}$. All of these wireless technologies operate on their own specified spectrum. To meet the growing demand for mobile wireless services, various approaches to integrate these networks for sharing resources between them have been introduced ${ }^{3}$.

Heterogeneous wireless network is one of these approaches which offer best services through best network at anytime and anywhere ${ }^{4}$.

To utilize the available radio frequency spectrum, cognitive radio is drawing much attention. Both licensed and unlicensed users can use the frequency spectrum using cognitive radio technique . $^{5}$

On a consequence, the spectrum sharing between WiFi and WiMAX is approached ${ }^{6}$.

A spectrum sharing method is $\operatorname{proposed}^{7}$, where $\mathrm{WiFi}$ access points (AP) within a WiMAX service area temporarily use the unused spectrum of WiMAX. According to Figure 1, there is a WiMAX service area covering by a WiMAX base station. As the service area is very wide (several kilometers) there are some WiFi APs within the service area. A Spectrum Manager (SM) collects the information about unused WiMAX spectrum, load on the WiFi APs and temporarily assigns the spectrum to WiFi APs.

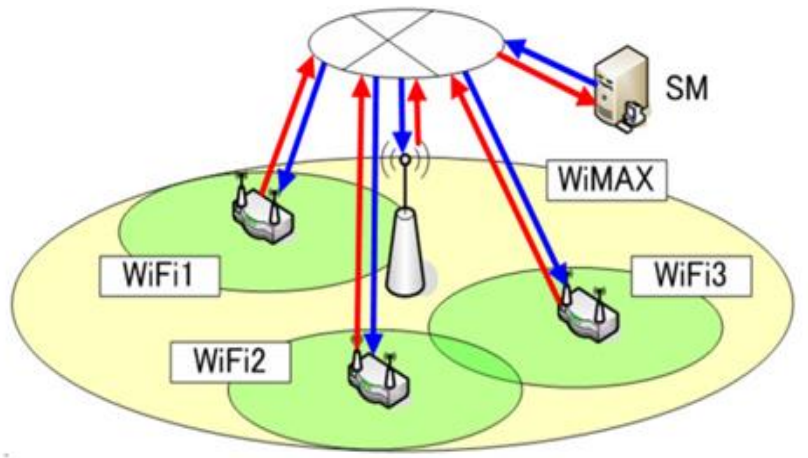

Fig. 1. Network model ${ }^{7}$

However, the SM can't assign the same spectrum to adjacent WiFi APs as it may cause interference problem. To overcome this problem, an efficient on-line spectrum sharing algorithm should be developed that can find out the optimum spectrum assignment pattern (most eligible $\mathrm{WiFi}$ APs for spectrum assignment) based on the load distribution (no of users connected to the WiFi APs) at that time.

A spectrum sharing algorithm is proposed ${ }^{7}$, which is based on Genetic Algorithm (GA). In this paper, the performance of the algorithm is evaluated. Also, an alternative method is developed based on Linear Programming (LP) for the search of optimum spectrum assignment pattern for comparative analysis. 


\section{Methodology}

\section{A. Spectrum sharing based on GA}

To find the optimum spectrum assignment pattern, in this method the sum of the load of WiFi APs was used as fitness value of GA. As, same band of WiMAX spectrum can't be shared to adjacent WiFi APs at the same time due to interference problem, this condition was used as constraint of $\mathrm{GA}^{7}$.

Let us assume $\mathrm{n}$ cells in an access area of a WiMAX BS as shown in Figure 2, where some of these cells contain WiFi APs. A spectrum assignment pattern can be prepared as Table 1, where the element ' 1 ' ' in a cell means the WiMAX spectrum can be assigned to the WiFi AP of the cell according to the pattern. This type of patterns has been applied as individuals in GA.

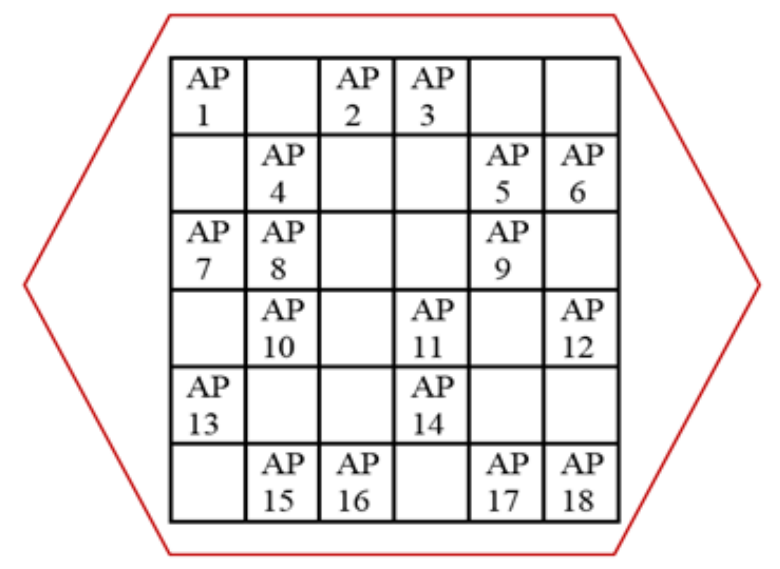

Fig. 2. WiMAX coverage areas with cells with and without WiFi AP

Table 1. A spectrum assignment pattern.

\begin{tabular}{|l|l|l|l|l|l|}
\hline 0 & 0 & 1 & 0 & 0 & 0 \\
\hline 0 & 0 & 0 & 0 & 0 & 1 \\
\hline 1 & 0 & 0 & 0 & 0 & 0 \\
\hline 0 & 0 & 0 & 1 & 0 & 0 \\
\hline 1 & 0 & 0 & 0 & 0 & 0 \\
\hline 0 & 0 & 1 & 0 & 1 & 0 \\
\hline
\end{tabular}

At the first step, 1 individuals (as Table 1) are generated randomly and the set of them are defined as initial group. Next, the fitness value of each individual are calculated using Equation 1 and the individual carrying highest fitness value is marked as candidate individual.

$$
E=\sum_{i=1}^{n} I_{i} Z_{i}
$$

Where, $Z_{i}=\frac{X_{i}}{Y_{i}}$ is the load of WiFi AP in cell $\mathrm{i}$ and $\mathrm{Xi} \& \mathrm{Yi}$ denote the number of connected users and the number of available spectra of the AP of cell i respectively.

At the next step, it makes pair-wise crossover between the individuals and calculates the fitness value of each reproduced individual. At this point the number of individuals becomes larger than the initial group. Hence, a selection process is executed to keep the number of individual as many as initial group. In this step, roulette selection is introduced and the survival probability is calculated using Equation 2.

$$
\boldsymbol{p}_{j}=\frac{E_{j}}{\sum_{j=1}^{l+2 m} E_{j}}
$$

Where, $\mathrm{m}$ is the number of parents participated in crossover. Among the $1+2 \mathrm{~m}$ individuals, 1 individuals containing higher $p_{j}$ value than others are selected.

However, if all the individuals are identical, any new assignment pattern can't be generated after crossover. Hence, a mutation process is executed if all the individuals are identical. After a certain number of iteration, the individual with highest fitness value is considered as the optimum assignment pattern and the WiMAX spectrum is assigned according to the pattern.



Fig. 3. Flowchart of the Spectrum sharing algorithm based on GA. 


\section{B. Developed LP model}

As an alternative approach to find the optimum spectrum assignment pattern, a LP model is developed in this paper.

To find an optimum solution, the problem is expressed as the ideal form of linear programming, where the objective function and the constraints are represented as linear function of decision variables.

Let define the decision variables as $\mathrm{x}_{1}, \mathrm{x}_{2}, \ldots \mathrm{x}_{\mathrm{n}}$, where they take value of ' 0 ' or ' 1 ' (binary variable) and they represent $\mathrm{AP}_{1}, \mathrm{AP}_{2}, \ldots \mathrm{AP}_{\mathrm{n}}$ respectively.

As the sum of the load of Wi-Fi APs is used as the fitness value for GA, it is also used as objective function for the LP model. The objective function is represented as linear function of decision variable as Equation 3.

$$
E=\sum_{i=1}^{n} Z_{i} \mathrm{x}_{\mathrm{i}}
$$

Where, $\mathrm{Zi}=\mathrm{Xi} / \mathrm{Yi}$ is the load of the $\mathrm{i}$-th WiFi AP.

Since at the same time the same spectrum is disallowed to assign to the adjacent Wi-Fi APs, this is used as constraint of this problem. To represent the constraint in terms of linear equations we proceed as follows.

Let assume the AP deployment pattern as Figure 2. According to the pattern if we assign same spectrum to $\mathrm{AP}_{1}$ and $\mathrm{AP}_{4}$ radio interference will occur. This constraint can be represented by a linear equation as Equation 4.

$$
x_{1}+x_{4} \leq 1
$$

Where, $\mathrm{x}_{1}$ and $\mathrm{x}_{4}$ are decision variables of $\mathrm{AP}_{1}$ and $\mathrm{AP}_{4}$ respectively.

Similarly to maintain the constraint between $\mathrm{AP}_{14}$ and $\mathrm{AP}_{17}$ the linear constraint equation is as Equation 5.

$$
x_{14}+x_{17} \leq 1
$$

Where, $x_{1}$ and $x_{4}$ are decision variables of $\mathrm{AP}_{1}$ and $\mathrm{AP}_{4}$ respectively.

At the same way all the possible constraint equations can be implemented. After representing the problem as described above, various algorithms (i.e. Simplex algorithm, Karmarker's algorithm) can be used to find the optimum assignment pattern ${ }^{8}$.

\section{Simulation}

To evaluate the performance of the both spectrum sharing algorithm (GA and LP) a Wi-Fi/WiMAX integrated network has been designed using OPNET Modeler 14.5A (a network design and simulation software) ${ }^{9}$.

One WiMAX BS covering a hexagonal area with $6 \times 6=36$ square cells into the coverage area where randomly selected 18 cells have WiFi AP was set as the network model which is shown in Figure 4.

All the WiFi APs and the WiMAX BS were connected to an FTP server through a gateway switch. Users are supported to download files (BE traffic) from the FTP server by connecting to AP (when they are at the cell with AP) or WiMAX BS (when they are at the cell without AP). Users were randomly distributed over the whole network area. Since WiFi APs were set up in places where people gather (e.g. cafe, office, hall etc.), more users were distributed at the cells with Wi-Fi APs than without APs.

The spectrum of the WiMAX BS was divided into several channels each having width of $20 \mathrm{MHz}$. On the other hand the spectrum of the WiFi was divided into three channels of $22 \mathrm{MHz}$ in $2.4 \mathrm{GHz}$ ISM band.

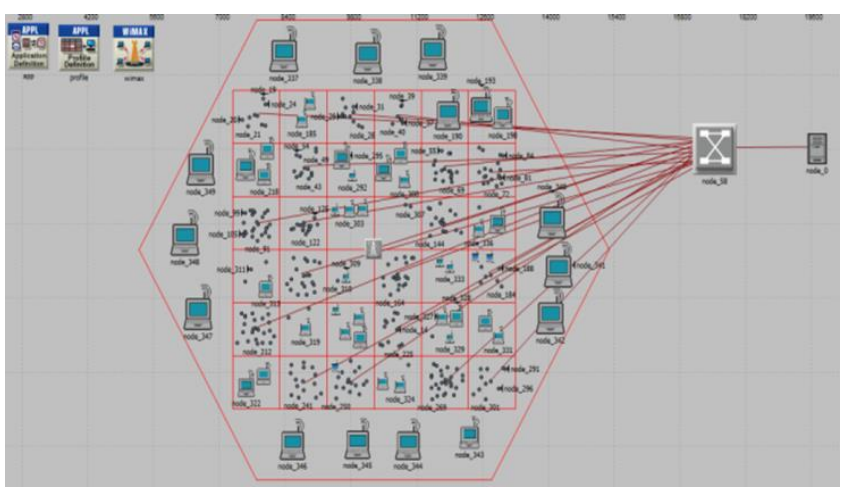

Fig. 4. A scenario of designed WiMAX/WiFi integrated network.

Table 2 and Table 3 show the WiMAX and WiFi simulation parameters respectively which were set at the network.

Table 2. WiMAX simulation parameters

\begin{tabular}{|c|c|}
\hline Simulation Parameter & Value \\
\hline Service Class Name & Bronze \\
\hline Scheduling Type & Best Effort (BE) \\
\hline Maximum Latency & $30 \mathrm{~ms}$ \\
\hline PHY Profile & OFDM \\
\hline Frame Duration & $5 \mathrm{~ms}$ \\
\hline Duplexing Technique & TDD \\
\hline Antenna Gain & $15 \mathrm{~dB}$ \\
\hline Maximum Number of SS nodes at BS & 100 \\
\hline Maximum Transmit Power & 0.5 \\
\hline Modulation and Coding & $64-\mathrm{QAM} \mathbf{1} 2$ \\
\hline Multipath Channel Model & ITU Pedestrian A \\
\hline Terrain Type & Flat \\
\hline
\end{tabular}

Table 3. WiFi simulation parameters

\begin{tabular}{|c|c|}
\hline Simulation Parameter & Value \\
\hline Physical Characteristics & Direct Sequence \\
\hline Data Rate & $11 \mathrm{Mbps}$ \\
\hline Transmit Power & $0.005 \mathrm{~W}$ \\
\hline Packet Reception-Power Threshold & $-95 \mathrm{dBm}$ \\
\hline AP-Beacon Interval & $0.02 \mathrm{~s}$ \\
\hline Max Receive Lifetime & $0.5 \mathrm{~s}$ \\
\hline Buffer Size (Bits) & 256000 \\
\hline CTS-to-Self Option & Enabled \\
\hline
\end{tabular}




\section{Results}

To evaluate the performance of both of the methods, average ftp download response time (s), average ftp traffic received $(\mathrm{KB} / \mathrm{s})$, and $\mathrm{Wi}-\mathrm{Fi}$ throughput $(\mathrm{Mb} / \mathrm{s})$ was selected as performance parameter.

Figure 5, $6 \& 7$ shows the average ftp download response time (s), average ftp traffic received $(\mathrm{KB} / \mathrm{s})$, and $\mathrm{Wi}-\mathrm{Fi}$ throughput $(\mathrm{Mb} / \mathrm{s})$ as function of simulation time respectively for without spectrum sharing, spectrum sharing using GA and spectrum sharing using developed LP model.

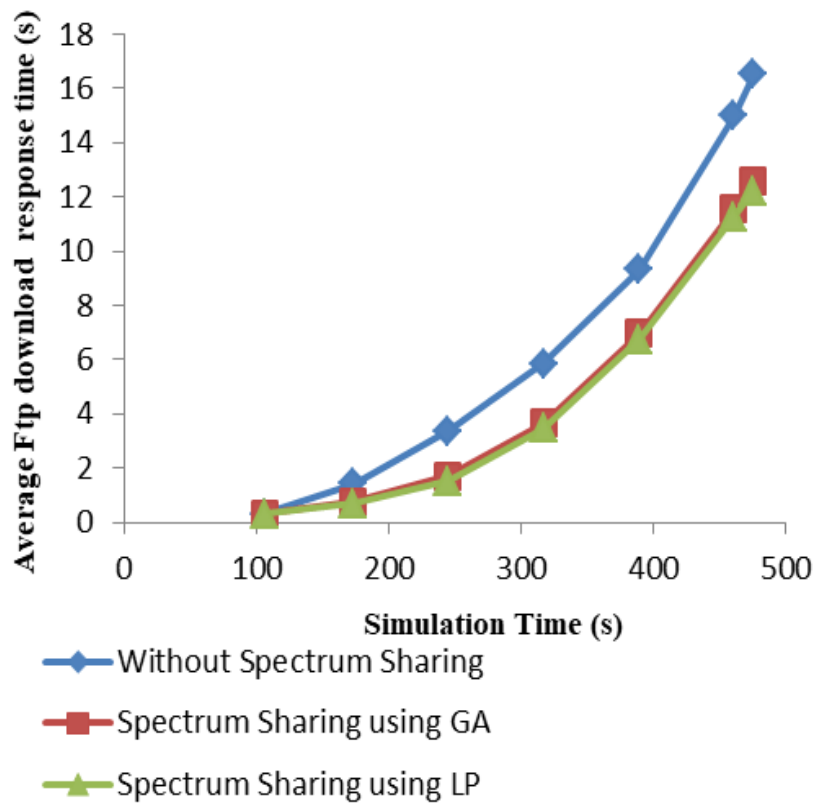

Fig. 5. Average ftp download response time vs. simulation time for spectrum sharing using GA \& LP and without spectrum sharing.

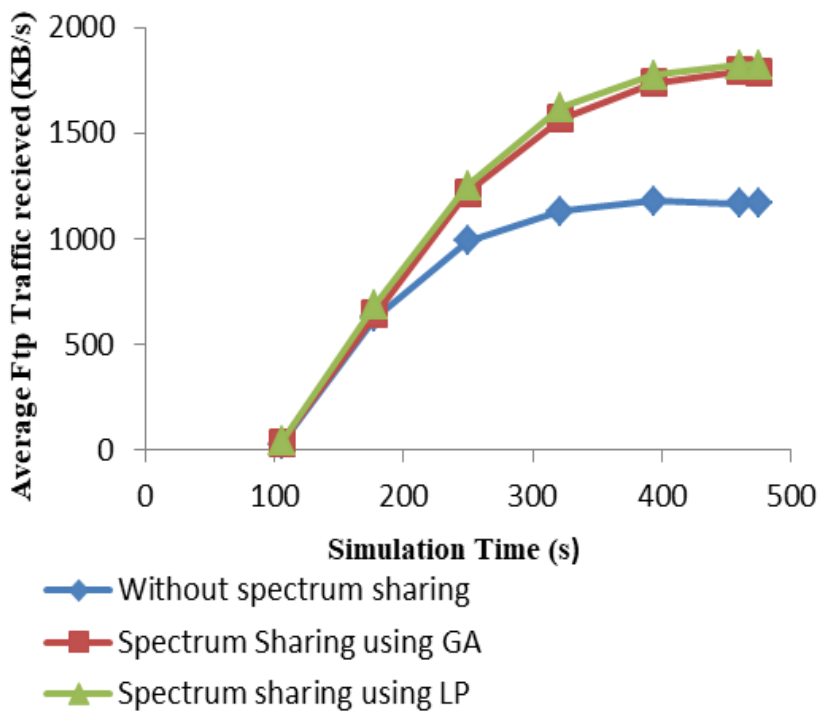

Fig. 6. Average ftp traffic received (KB/s) vs. simulation time for spectrum sharing using GA \& LP and without spectrum sharing.

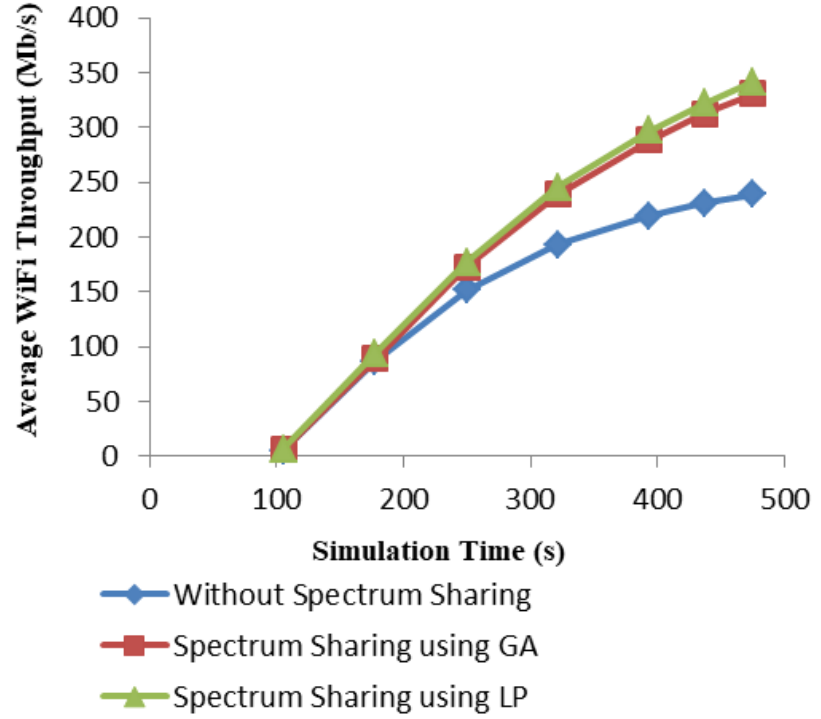

Fig. 7. Average ftp $\mathrm{WiFi}$ throughput $(\mathrm{Mb} / \mathrm{s})$ vs. simulation time for spectrum sharing using GA \& LP and without spectrum sharing.

As the traffic generation rate increases exponentially with simulation time, the download response time is increased accordingly (Figure 5).

However, the result shows that two methods (GA and LP) with spectrum sharing shorten the download response time, increased the average traffic received and average $\mathrm{WiFi}$ throughput compared with the method without spectrum sharing. This is because the capacity of the integrated network was improved by allocating unused spectrum of WiMAX BS to Wi-Fi AP. It can be noticed that the performance of GA and LP is almost same in terms of download response time. However, the developed LP model shows moderately better performance in the case of average traffic received $(\mathrm{KB} / \mathrm{s})$ and average $\mathrm{WiFi}$ throughput $(\mathrm{Mb} / \mathrm{s})$ (Figure 6 and Figure 7) though it was found during the simulation that, LP model takes larger time to reach the optimal solution.

\section{Conclusion}

In this work, for the purpose of using spectrum more efficiently in WiMAX/WiFi integrated network the performance of the method of spectrum assignment based on GA has been evaluated. Also, a LP model has been developed as an alternative method. To evaluate the performance, the average ftp download response time (s), average received traffic $(\mathrm{KB} / \mathrm{s})$ and $\mathrm{WiFi}$ throughput $(\mathrm{Mb} / \mathrm{s})$ was selected as performance parameter.

The simulation result shows that, both the methods (GA and LP) report substantially better performance than the case when no spectrum is shared between WiFi and WiMAX. However, the developed LP model gives slightly better performance than GA but it takes larger time to reach optimal solution.

However, on-line efficiency of the methods is very important aspect for evaluating the performance since any 
methods of spectrum sharing works in on-line. As, GA is a population based optimization algorithm and it involves huge computations, it will obviously face challenges to provide optimum solution within very short time. To overcome this challenge, in this paper, the number of iteration of GA was limited so that, it can provide a solution within a reasonable time and the optimality of the solution is justified by the simulation results.

Another important point is that, the computational burden of GA may challenge the application of the method in single processor based computing system. However, as the algorithm will be implemented by the SM in the integrated network, implementation of highly efficient processor based computing system in SM can overwhelm the challenge.

\section{References}

1. Tragos, E.Z., S. Zeadally, A.G. Fragkiadakis, and V.A. Siris, 2013. Spectrum assignment in cognitive radio networks: A comprehensive survey. IEEE Communications Surveys \& Tutorials, 15(3), 1108-1135.

2. Varshney, U., 2012. 4G wireless networks. IT Professional, 14(5), 34-39.
3. Song, W., W. Zhuang and Y. Cheng, 2007. Load balancing for cellular/WLAN integrated networks. IEEE network, 21(1), 27-33.

4. Das, S., M.J. Rashid, and S.K. Aditya, 2010. Fuzzy Logic Based Adaptive Handoff Algorithm for Heterogeneous Network. Dhaka Univ. J. Sci., 58(1), 113-117.

5. Tsiropoulos, G.I., O.A. Dobre, M.H. Ahmed and K.E. Baddour, 2016. Radio resource allocation techniques for efficient spectrum access in cognitive radio networks. IEEE Communications Surveys \& Tutorials, 18(1), 824-847.

6. Andrews, N., Y. Kondareddy, and P. Agrawal, 2010. Prioritized resource sharing in WiMax and WiFi integrated networks. In Wireless communications and networking conference (WCNC), 2010 IEEE. 1-6.

7. Kinoshita, K., M. Nakagawa, K. Kawano, and K. Murakami, 2014. An Efficient Spectrum Sharing Method Based on Genetic Algorithm in Heterogeneous Wireless Network. International Journal of Computer Networks \& Communications, 6(5), 145.

8. Karloff, H., 2008. Linear programming. Springer Science \& Business Media.

9. OPNET Modeler, http://www.opnet.com/. 
